\title{
Five observations concerning the intended meaning of the intuitionistic logical constants
}

\author{
Gustavo Fernández Díez \\ University of Murcia \\ Facultad de Filosofía \\ Apartado 4021 \\ E-30071, Murcia (Spain) \\ gfdezdp@fcu.um.es
}

\begin{abstract}
This paper contains five observations concerning the intended meaning of the intuitionistic logical constants: (1) if the explanations of this meaning are to be based on a non-decidable concept, that concept should not be that of 'proof'; (2) Kreisel's explanations using extra clauses can be significantly simplified; (3) the impredicativity of the definition of $\rightarrow$ can be easily and safely ameliorated; (4) the definition of $\rightarrow$ in terms of 'proofs from premises' results in a loss of the inductive character of the definitions of $\vee$ and $\exists$; and (5) the same occurs with the definition of $\forall$ in terms of 'proofs with free variables'.

KEY WORDS: 'Intuitionistic semantics', 'logical constant', 'construction', 'proof', 'intended meaning'.
\end{abstract}

\section{Introduction}

The attempts to make explicit the intended meaning of the intuitionistic logical constants have lead to a number of 'informal explanations'. The accuracy of these explanations is not very important for the actual practice of intuitionistic mathematics, which relies mainly on intuitive understanding and previous usage, but it is essential from a philosophical point of view:

" $(. .$.$) it is therefore necessary, in the first place, to inquire whether$ these explanations of the logical constants are coherent or not, whether they confer intelligible meanings on them; if this question $(. .$. has to be answered negatively, the whole conception, inherent in intuitionistic mathematics, of how mathematical statements are to be given meaning will have been shown to be defective." (Dummett (1977): 390.)

The idea of how such explanations should look like is perhaps best represented by the usual definition of intuitionistic disjunction: 
Definition 1 A proof of $p \vee q$ is either a proof of $p$ or a proof of $q$.

This definition combines three qualities which make it particularly attractive: (a) it is very simple; (b) it makes transparent that aspect of the intuitionistic $\checkmark$ which constitutes its most salient difference with its classical counterpart (indeed, in classical mathematics we often prove $p \vee q$ without proving either $p$ or $q$ ); and (c) it has an 'inductive character', that is: the meaning of a complex sentence is given in terms of the meanings of its constituents, sentences logically simpler than it.

Unfortunately, when we try to reduce the other logical constants - and in particular $\rightarrow$, $\neg$ and $\forall-$ to an schema such as Definition 1, we encounter serious difficulties. In the present paper I shall make five observations concerning these difficulties and the attempts that have been made to overcome them.

\section{The conditional}

I would like to start by looking at the definition of the intuitionistic conditional. Of the various versions which currently appear in the literature, the following is one of the simplest, taken from a well-known textbook on constructivism:

Definition 2 A proof of $p \rightarrow q$ is a construction which permits us to transform any proof of $p$ into a proof of $q$.

(Troelstra and van Dalen (1988): 9. In this and the subsequent quotations I always use my choice of notation.)

This definition resembles the previous one for intuitionistic disjunction, but it is based on a peculiar appeal to any - arbitrary-proof of $p$, and this fact makes it defective in two ways that the other is not. In the first place, it is impredicative: the construction which is being defined and which proves $p \rightarrow q$ must be able to transform any possible proof of $p$ into a proof of $q$; as no boundary is put on the complexity of those possible proofs of $p$, they could include some complicated roundabout proofs which involved reference to the sentence $p \rightarrow q$ itself, and hence to the same proof being defined. In sum: the definition of a proof of $p \rightarrow q$ appeals to a totality of proofs, with some of which the very proof of $p \rightarrow q$ could be intimately related.

In the second place, this definition of $\rightarrow$ has the effect of converting the proof relation induced into a non-decidable relation. Indeed, it is quite possible that a given construction $c$ is able to transform every proof of $p$ into a proof of $q$, but without being obviously so; and in such a case the fact that $c$ does transform every proof of $p$ into a proof of $q$ will ask for a separate proof. As long as we cannot provide this additional proof, we will be unable to decide whether, according to Definition 2, the original construction $c$ is a proof of $p \rightarrow q$ or not.

However, it is extremely unnatural - both intuitionistically and classicallyto say that a construction might be a 'proof' of a given sentence, but that we cannot decide whether that is the case or not; and that we need a further proof, 
which requires true ingenuity to find, in order to establish that $c$ is indeed the 'proof' of the sentence in question.

I shall examine the undecidability problem first, and leave the impredicativity, less urgent one, for later.

\section{A fundamental dichotomy}

The fact that Definition 2 induces a non-decidable relation is only a particular case of a general dichotomy, familiar from computer programming: the dichotomy between a concrete detailed mathematical construction which performs a certain task, and that additional construction which proves that the first one does indeed the task in question. Very often we define tentatively a mathematical construction with the purpose of operating in a particular way, but once the construction is completely defined, it is not at all obvious that it will always behave in the way it was intended to. In such cases we must devise a separate proof that our completed construction does the job required, and the resulting proof is a different object from the original construction itself.

Sundholm has stressed the distinction between a mathematical construction as a finished object, and the act or process of constructing it (a distinction embedded in Martin-Löf's type theory, cf. Sundholm (1983): 164-168, (1994): 144-148, Martin-Löf (1984), (1994) and Nordström, Petersson and Smith (1990)). Moreover, Sundholm maintains that:

"The proof that the construction does what it is supposed to do is not itself a construction object, but rather the act of proof/construction whereby the construction object is given." (Sundholm (1994): 148, footnote.)

"For any proposition, be it an implication, a conjunction or what have you, it must be possible to recognise its proof-objects as such (...). This property has to be ensured through the way in which the relevant proof-object is given (the act of proof/construction)." (Sundholm (1994): 148, his italics.)

However, it seems to me that this cannot be always the case. For example, let us consider a construction, $c$, which enables us to find, for each positive integer $n$, a prime number bigger than $n$ which is of the form $4 m-1$ for some $m$ (a special case of Dirichlet's theorem):

take the smallest divisor of $4 n !-1$ which is of the form $4 m-1$ for some integer $m$.

It is very easy to prove that $c$ indeed does the job required, i.e.: that the result of applying $c$ to a positive integer $n$ is always a number which is prime, bigger than $n$ and of the form $4 m-1$ for some $m$. But this fact is not completely obvious, and the mere description of $c$ does not reveal it: the proof which establishes it is a very different construction from $c$ itself. 
Even further, sometimes an open mathematical problem remains unsolved, not because we lack the basic construction which does or is supposed to do the job required, but because we lack the proof that such a construction does indeed that job. An example is Goldbach's conjecture: the construction for decomposing an arbitrary even number $n$ into a sum of two primes, is a matter of finite routine searching over the numbers smaller than $n$. Insofar as it has been possible to test such a construction, it worked; but we cannot prove that it will work in general.

Hence we have a very simple construction which appears to transform all proofs of ' $n$ is even' into proofs of ' $n$ is a sum of two primes' (showing two such primes), but we cannot decide whether it does so in general, for all natural $n$, and because of that we cannot decide whether it will be, according to Definition 2 , a proof of 'if $n$ is even then $n$ is a sum of two primes' for every $n$.

Such a situation, by the way, is quite different from those others where we even lack the basic construction which is supposed to do the job required. For example, in the case of the twin prime conjecture, we simply do not have any candidate for a construction which appeared to transform any positive integer into a pair of twin primes bigger than it, and behaved so, e.g., when tested against relatively small numbers.

\section{Kreisel's extra clauses}

In order to ensure the decidability of the proof-relation induced, Kreisel proposed the introduction of an 'extra clause' in the definition:

Definition $3 A$ proof of $p \rightarrow q$ is a pair of constructions $\left(c_{1}, c_{2}\right)$, where $c_{1}$ proves that $c_{2}$ transforms any proof of $p$ into a proof of $q$.

(cf. Kreisel (1961): 107, footnote, (1962): 205, (1965): 128). Hence, if we have a construction which appears to transform all proofs of $p$ into proofs of $q$, but we are unable to prove this fact, we can readily conclude that we $d o$ not, according to this definition, have a proof of $p \rightarrow q$.

In turn, Kreisel's definition is not inductive: the proof of $p \rightarrow q$ is defined not only in terms of proofs of $p$ and $q$, but also of an extra proof, $c_{1}$, of a much wider scope. This loss of the inductive character reduces significantly the explanatory power of the definition.

At first, Kreisel's extra clauses were received largely as a straight improvement (cf. e.g. Troelstra (1969): 5, (1977): 977, van Dalen (1973): 24, or Dummett (1977): 399); but after some debate (e.g. Prawitz (1977): 27, Sundholm (1983): 153-161, Weinstein (1983): 263-266), a number of authors abandoned them, reverting henceforth to simpler formulations such as Definition 2 (e.g. van Dalen (1983): 166, (1986): 231, Troelstra and van Dalen (1988): 9):

"It must be pointed out however that the decidability of the proofrelations has been critized and that the 'extra clauses' are not universally accepted." (van Dalen (1986): 232.) 
This debate was strongly influenced by the idea that the constructive meaning of mathematical sentences has to be given in terms of proof conditions as opposed to truth conditions, an idea which is deeply rooted among intuitionists (Heyting (1956): 97, Kreisel (1962): 201, Dummett (1977): 12). However, it seems to me that, if we want to dispense with Kreisel's extra clauses and preserve the inductive structure of the definition, then we must change the concept of 'proof', at the core of that inductive structure, and replace it by a different concept.

\section{The operational interpretation}

As a matter of fact, it is not difficult to find such a replacement. For example, we could say that a construction $c$ 'performs' a given sentence $p$ when the application of $c$ carries out those constructive operations that the sentence $p$ claims to be possible. Thus, if $p$ says 'every positive integer has a bigger prime of the form $4 m-1$ ', then the application of the corresponding construction $c$ to a positive integer $n$ should always yield a number which is prime, bigger than $n$ and of the form $4 m-1$ for some $m$. This type of interpretation is very similar - if not identical - to Kleene's concept of realizability, but I shall call it here 'the operational interpretation'.

In the case of an intuitionistic conditional, this definition would read:

Definition $4 A$ construction c performs $p \rightarrow q$ when it transforms every construction $c$ which performs $p$ into a construction which performs $q$.

This clause has exactly the same structure as Definition 2, and the relation induced, which in this case is the 'performing relation', is indeed a non-decidable relation as well. However, in the present case there is no paradox in the fact that the performing relation turns out to be undecidable. As a matter of fact, here we draw a clear distinction between the construction $c$ which performs $p \rightarrow q$, and a proof that could be supplied, showing that $c$ in effect performs $p \rightarrow q$.

Whether it is intuitionistically acceptable to have a non-decidable concept at the core of the semantic definition, is a very deep issue which I cannot attempt to settle here. I do not even have a determinate opinion myself. My contention is (observation number 1 ) that if we choose to have a non-decidable concept, in order to preserve the inductive structure of the semantic definition-as some authors do-, then such a concept should not be that of 'proof', but one more according to its non-decidable character.

\section{$6 \quad$ Kreisel's interpretation revisited}

Besides, the operational interpretation can help us to simplify Kreisel's definition of the conditional in a significant way: according to Kreisel's interpretation, in a proof $\left(b_{1}, b_{2}\right)$ of a nested conditional sentence such as

$$
(p \rightarrow q) \rightarrow(r \rightarrow s)
$$


the second component $b_{2}$ is required to transform every pair $\left(c_{1}, c_{2}\right)$ which proves $p \rightarrow q$ into a corresponding pair $\left(d_{1}, d_{2}\right)$ which proves $r \rightarrow s$. But a moment's reflection shows that, as a matter of fact, it is enough that $b_{2}$ transforms every construction which performs $p \rightarrow q$ into a construction which performs $r \rightarrow$ $s$. And then, if the extra clause $b_{1}$ proves that this is really the case, the transformation of the other extra clauses will follow quite trivially.

Indeed, let us suppose that $b_{2}$ transforms every construction which performs $p \rightarrow q$ into a construction which performs $r \rightarrow s$; and let $b_{1}$ be a proof of this fact. Let us also assume that $p, q, r$ and $s$ are all atomic sentences, so that there is no need of further extra proofs: if a construction performs $p, q, r$ or $s$, this fact must be apparent, so it immediately qualifies as a proof of it by all standards.

Then, if we are supplied with a 'full' proof $\left(c_{1}, c_{2}\right)$ of $p \rightarrow q$, we can use $b_{2}$ to transform $c_{2}$ into a construction $d_{2}=b_{2}\left(c_{2}\right)$ which performs $r \rightarrow s$. And the proof that $d_{2}$ is indeed such a construction will follow at once from $c_{1}$, which ensures that $c_{2}$ performs $p \rightarrow q$, and our previous proof $b_{1}$, which ensures that the result of applying $b_{2}$ to a construction performing $p \rightarrow q$ is a construction performing $r \rightarrow s$.

This suggests (observation number 2) that Kreisel's requirement of the extra clauses is not in essence an inductive requirement, which demands to be placed at each step of the inductive definition, but rather, a direct one, which demands to be placed on top of a previous definition, such as the operational interpretation, which is itself inductive.

This observation was inspired to me by a question posed on this respect, long ago, by Kreisel himself:

"There is an additional distinction which has so far not been formally necessary, but which is probably important, for example in the explanation of implication (or universal quantification). When we think of the pair $\left(b_{1}, b_{2}\right)$

$b_{1}$ proves the identity: for variable $c$, if $c$ proves $p$, then $b_{2}(c)$ proves $q$,

$b_{2}$ is a genuine function or operation, while $b_{1}$ recognizes that $b_{2}$ satisfies the condition stated: thus $b_{1}$ is a judgement. But similarly, since in general both the arguments $c$ and the values $b_{2}(c)$ of $b_{2}$ are such pairs, say $c=\left(c_{1}, c_{2}\right)$ and $b_{2}(c)=\left(d_{1}, d_{2}\right)$, should the function $d_{2}$ depend both on $c_{2}$ and $c_{1}$ (or only on $c_{2}$ )?" (Kreisel (1970): 145-146, endnote.)

He does not give an explicit answer to his own question, nor has he developed this point later - at least not to my knowledge.

If I am correct - in which case the answer to Kreisel's question is definitely 'only on $c_{2}$ '-, the natural way to re-formulate Kreisel's definition of the conditional would be: 
Definition 5 A proof of $p \rightarrow q$ is a pair of constructions $\left(c_{1}, c_{2}\right)$, where $c_{1}$ proves that $c_{2}$ performs $p \rightarrow q$.

Or more in general,

Definition 6 A proof of a sentence $p$ is a pair of constructions $\left(c_{1}, c_{2}\right)$, where $c_{1}$ proves that $c_{2}$ performs $p$.

\section{The impredicativity of $\rightarrow$}

It is time to go back to the impredicativity problem. We noticed that the definition of a proof of $p \rightarrow q$ referred to arbitrary proofs of $p$, and that some of those proofs could be roundabout proofs, in which the very proof of $p \rightarrow q$ played a role. This problems does concern Kreisel's definition, and the operational interpretation as well, insofar as there could be roundabout constructions which perform $p$ on the basis of a mid-step performance of $p \rightarrow q$.

The problem is substantially ameliorated, however, if we consider that in virtually all intuitionistic proofs of conditional sentences such as $p \rightarrow q$, the only property of the possible proofs of $p$ which is employed in the derivation of $q$ is precisely that of being a proof of $p$. Hence, the transformation of the proofs of $p$ does not depend on the internal structure of those proofs, except for the requirements that the inductive definition places on them, according to the logical form of $p$ (for example, if $p$ is a disjunction, then any proof of $p$ must include a proof of one of the disjuncts, and the subsequent proof of $q$ might very well depend on which disjunct is the one included).

However, as it happens, the only notable occasion in which all possible proofs of the antecedent were classified and transformed according to its internal structure and beyond the requirements that the inductive definition places on them, is Brouwer's attempted proof of the bar theorem, which as we know is incorrect and no way has been found to correct it preserving its original form (cf. e.g. Brouwer (1927), and for a discussion Dummett (1977): 94-104).

This suggests (observation number 3) a very simple way in which we could safely ameliorate the impredicativity from our two previous explanations of the intuitionistic conditional: to replace the occurrence of the word 'transforms' (or 'transform') in each of them, by e.g. 'extends' ('extend'). And the fact that the intuitionistic conditional has never been successfully used, in mathematical practice, in other but this restricted sense, probably means that such is the best way to characterize its intended interpretation.

In fact, Heyting's original definitions of the intuitionistic conditional were very much on these lines:

" $p \rightarrow q$ represents then the intention of a construction which, from each demonstration of $p$, leads to a demonstration of $q . "$ (Heyting (1934): 17.)

"The implication $p \rightarrow q$ can be asserted, if and only if we possess a construction $c$, which, joined to any construction proving $p$ 
(supposing that the latter be effected), would automatically effect a construction proving $q$. In other words, a proof of $p$, together with $c$, would form a proof of q." (Heyting (1956): 98. My italics in 'joined'.)

\section{Proofs from premises}

A yet further refinement on the previous proposal is to define a proof of a conditional sentence $p \rightarrow q$ directly as a 'proof of $p$ with premise - or hypothesis - $q$ '. This idea is in fact the oldest, as it corresponds to Kolmogorov's interpretation of the intuitionistic conditional in terms of mathematical problems:

"(..) $p \rightarrow q$ is the problem of 'solving the problem $q$, supposing that the solution to $p$ is given'." (Kolmogorov (1932): 59.)

The same idea is the motivation behind Gentzen's rule of $\rightarrow$-introduction (which he considered a meaning definition, cf. Gentzen (1935): 78-80), and has been treated since then as a faithful explanation of the meaning of the intuitionistic conditional e.g. by Sundholm (1986): 490, Martin-Löf (1985): 45, (1987): 410412, or Bridges and Richman: "the statement $p \rightarrow q$ means that $q$ holds under the assumption that $p$ holds" (Bridges and Richman (1987): 11).

The idiosyncrasies of this type of interpretation have been widely ignored, but it is quite remarkable that, prima facie, under this definition the decidability problem seems to disappear completely: indeed, there is no reason in principle why we should not be able to recognize a 'proof of $q$ with premise $p$ ' when we see one, provided that we already know the intuitionistic meanings of $p$ and $q$.

However, this gain of decidability is again not without cost. To start with, once we agree to define the proof of any conditional sentence $p \rightarrow q$ as a proof of $q$ with premise $p$, we must immediately re-define all the other logical constants in terms of a finite set of premises. For instance, in the case of a sentence such as

$$
p \rightarrow(r \rightarrow(s \vee t))
$$

the proof of the disjunction $s \vee t$ should be given in terms of the two premises $p$ and $r$.

But when we try to carry out such a re-definition, we run into the most unexpected problem. Indeed, we cannot admit the definition:

Definition 7 A proof of $p \vee q$ from a set $\mathcal{P}$ of premises is either a proof of $p$ from premises $\mathcal{P}$, or a proof of $q$ from premises $\mathcal{P}$,

because that would legitimate the inference

$$
r \rightarrow(s \vee t) \vdash(r \rightarrow s) \vee(r \rightarrow t),
$$

which is not intuitionistically valid (i.e. it is not deducible in the intuitionistic propositional calculus).

Then, the only possible alternative seems to be: 
Definition 8 A proof of $p \vee q$ from a set $\mathcal{P}$ of premises is a proof from premises $\mathcal{P}$ that either a proof of $p$ or a proof of $q$ can be constructed.

But this definition is not inductive, as there is no decrease of logical complexity in the definiens.

Similarly, we cannot admit a definition of the intuitionistic existential quantifier such as:

Definition 9 A proof of $\exists x A(x)$ from a set $\mathcal{P}$ of premises consists in a construction $c$ in the domain plus a proof from premises $\mathcal{P}$ that $A(c)$,

because that would invalidate the intuitionistically accepted inference

$$
\forall x(A(x) \rightarrow B(x)) \vdash \exists x A(x) \rightarrow \exists x B(x) ;
$$

indeed, the transformation of a proof of $\exists x A(x)$ into a proof of $\exists x B(x)$ will in general depend on the particular object $c$ which the first proof provides as an instantiation (this point is made by Dummett (1977): 14-15, arguing against an explanation akin to Definition 9).

Then, the only possible alternative is:

Definition $10 A$ proof of $\exists x A(x)$ from a set $\mathcal{P}$ of premises is a proof from premises $\mathcal{P}$ that an object $c$ in the domain can be constructed, and a proof provided that $A(c)$,

which as before is not an inductive definition.

As we can see (observation number 4), although the appeal to 'proofs from premises' in the definition of the intuitionistic conditional appears to ensure the decidability of the proof relation, it has also the undesired effect of collapsing the inductive structure of the definition. This is similar to what happened with Kreisel's extra clauses, except for the striking fact that here the collapse takes place exactly in the definition of disjunction and the existential quantifier.

To be sure, it is easy to see that, in particular, the definition of the universal quantifier in terms of proofs from premises does not face the same problem; but for showing this we first have to examine the usual definition of the intuitionistic $\forall$.

\section{The universal quantifier}

Of the various definitions of the intuitionistic $\forall$ that can be found in the literature, the following is one of the simplest ones, analogue to Definition 2 for the conditional:

Definition 11 A proof of $\forall x A(x)$ is a construction which transforms any construction $c$ in the domain into a proof of $A(c)$.

If the domain of interpretation is non-decidable, this definition is supplemented accordingly: 
Definition 12 A proof of $\forall x A(x)$ is a construction which transforms any proof that a given construction $c$ belongs to the domain, into a proof of $A(c)$.

(cf. Troelstra and van Dalen (1988): 9). In either case, the definition induces a non-decidable relation, just as Definition 2 did, and admits either the introduction of a Kreisel's extra clause (which will make it decidable, but non-inductive), or, alternatively, the replacement of its central concept for one akin to 'performing' (which will preserve inductiveness but not decidability). The way to carry out both things is straightforward. And the simplification of Kreisel's definition on the basis of the operational interpretation (proposed in Section 5) can be straightforwardly extended to this case too.

Then, if we want to adapt this definition to the case of an arbitrary set of premises $\mathcal{P}$, the two available options:

Definition $13 A$ proof of $\forall x A(x)$ from premises $\mathcal{P}$ is a construction which transforms any construction $c$ in the domain into a proof of $A(c)$ from premises $\mathcal{P}$

and

Definition 14 A proof of $\forall x A(x)$ from premises $\mathcal{P}$ is a proof from premises $\mathcal{P}$ that we can transform any construction $c$ in the domain into a proof of $A(c)$,

are clearly equivalent in meaning. In particular, from a proof from premises $\mathcal{P}$ that we can transform each $c$ into a proof of $A(c)$, it is very easy to obtain a method which transforms each $c$ into a proof from premises $\mathcal{P}$ of $A(c)$ : all we have to do is to apply the previous proof to each given $c$. And the implication in the other direction is even more obvious.

This means that the definition of $\rightarrow$ in terms of proofs from premises results in a decidability problem for the subsequent clauses for $\vee$ and $\exists$, but not for that corresponding to $\forall$.

On the other hand, it is plain to see that formulations such as 13 and 14 do not, in turn, resolve the decidability problem inherent to the definition of $\forall$ itself. And the natural question is whether there is an analogous way to confront this problem

\section{Proofs with free variables}

The answer is 'yes': there is a most peculiar way which appears to preserve the decidability in the definition of $\forall$, apparently without inductive loss, and which runs entirely parallel to the appeal to proofs from premises in the case of the conditional:

Definition $15 A$ proof of $\forall x A(x)$ is a proof of $A(x)$ with free variable $x$.

This does not coincide with Kolmogorov's original formulation (which explicitly refers to a "general method for the solution of $A(x)$ for each particular value 
of $x$ ", cf. Kolmogorov (1932): 60), but it corresponds to Gentzen's rule of $\forall$ introduction (Gentzen (1935): 78), and can be found in other authors such as Sundholm (1986): 491 and Martin-Löf (1985): 54, (1987): 410-412.

Dummett has critized this definition on the grounds that it does not account for the soundness of the principle of mathematical induction: in general, a proof of $\forall x A(x)$ from $A(0)$ and $\forall x(A(x) \rightarrow A(x+1))$ will not be a free-variable proof of $A(x)$, but a method to transform each natural number $n$ into a proof of $A(n)$, the method being an iterating application of modus ponens (Dummett (1977): 14). However, this might be taken simply as evidence that induction is not a logical rule, which can be extracted right away from the intuitionistic meaning of the logical constants, but a genuinely mathematical principle.

In any case, the definition faces a problem completely analogous to that of its respective attempt for the conditional in terms of proofs from premises. To start with, it forces us to re-define all the other logical constants in terms of a finite set of free variables, something which has not been tried in detail before. But when we try to do it (observation number 5), we lose the inductive character of the corresponding definitions of disjunction and the existential quantifier.

Indeed, a proof of $\forall x(A(x) \vee B(x))$ will not be, in general, either a freevariable proof of $A(x)$ or a free-variable proof of $B(x)$, but rather, a free-variable proof of the disjunction $A(x) \vee B(x)$; and similarly, a proof of, say, $\forall x \exists y A(x, y)$, will not usually consist in the production of an object $c$ plus a proof of $A(x, c)$ with free variable $x$, but rather, in a free variable proof of $\exists y A(x, y)$.

On the other hand, it is important to notice that the definition of $\rightarrow$ in terms of 'proofs with free variables' (or in terms of 'proofs with premises and free variables') does not constitute any problem: in particular, from a given method to transform all free-variable proofs of $p$ into free-variable proofs of $q$, we can always obtain a 'uniform' free-variable proof of $p \rightarrow q$, the only (trivial) modification possibly needed being that if the free-variable proof of $p$ uses a particular sequence of free variables, the free-variable proof of $q$ which results after the application of the method is rearranged so that it uses exactly the same sequence. And the implication in the other direction is even more obvious.

\section{Atomic sentences and the rest}

The treatment of atomic sentences is easy, as intuitionistically we require that the basic properties of the interpretation are such that, for any given construction $c$ in the domain (plus a proof that $c$ belongs to it, if it is non-decidable), we already know how to recognize a proof $d$ that $c$ satisfies any of them. Hence $d$ will qualify as a proof of the corresponding sentence, and at the same time as a construction which performs it. And similarly for relations and functions.

The intuitionistic negation is defined as a conditional sentence which has as consequent a basic self-evident absurdity (a construction which is obviously impossible to carry out), and as antecedent the sentence negated. Hence all that has been said about the conditional applies to negation directly.

Conjunction is a straight 'sum' of the proofs (or performing constructions) 
corresponding to each conjunct: its definition does not create any problem at all.

Next, the explanation of the intuitionistic disjunction, which has been already touched on (Definition 1), does not pose a decidability problem, nor does it require the introduction of Kreisel's extra clauses, unless it is broaden so as to read, e.g.:

Definition 16 A proof of $p \vee q$ is either a proof of $p$, or a proof of $q$, or an effective procedure which enables us to find either of them,

in which case it will stand in a very similar position to the definition of the conditional.

The definition of the intuitionistic existential quantifier has also been mentioned, indirectly. It resembles that of the disjunction in many aspects. It is usually given as:

Definition 17 A proof of $\exists x A(x)$ is a pair of constructions $\left(c_{1}, c_{2}\right)$, where $c_{2}$ is a construction in the domain and $c_{1}$ proves $A\left(c_{2}\right)$.

Or, if the domain of interpretation is non-decidable:

Definition 18 A proof of $\exists x A(x)$ is a triple of constructions $\left(c_{0}, c_{1}, c_{2}\right)$, where $c_{0}$ proves that $c_{2}$ belongs to the domain, and $c_{1}$ proves $A\left(c_{2}\right)$.

In either case, it does not pose a decidability problem, unless we permit, as before, that an effective procedure for finding a pair $\left(c_{1}, c_{2}\right)$ or a triple $\left(c_{0}, c_{1}, c_{2}\right)$ directly qualifies as a proof of $\exists x A(x)$.

And finally, the way to extend the definition of disjunction to the operational interpretation is straightforward:

Definition 19 A construction performs $p \vee q$ when either it performs $p$ or it performs $q$.

And as for the existential quantifier, it is in the spirit of the operational interpretation that the reference to $c_{1}$ is eliminated:

Definition $20 A$ construction c performs $\exists x A(x)$ when some other construction performs $A(c)$;

and thus a proof that $c$ performs $\exists x A(x)$ would require the production of a concrete construction $d$ which performed $A(c)$.

We can now see that the two groups of intuitionistic logical constants: $\{\rightarrow, \neg, \forall\}$ and $\{\vee, \exists\}$ are really opposed to each other: $\rightarrow, \neg$ and $\forall$ pose a decidability problem, which $\vee$ and $\exists$ normally do not; and the attempts to resolve it produce a loss of inductiveness either in the definitions of $\rightarrow, \neg$, and $\forall$, or in those of $\vee$ and $\exists$.

What conclusion, if any, can be drawn from this last-unnumbered-observation, I leave to others to decide. 


\section{Acknowledgements}

The contents of this paper, in a somewhat different form, are part of my doctoral dissertation at the London School of Economics, written under the direction of Professor Colin Howson, and with the unofficial - but invaluable - advice of Professor Moshé Machover. The research was supported, at various stages, by the British Council, the British Academy, the Spanish Ministry of Education and Caja de Ahorros del Mediterráneo. I would like to thank as well an anonymous referee of the Journal of Philosophical Logic, for helpful comments and suggestions on an earlier draft.

\section{References}

Bridges, D. S. and R. Richman (1987): Varieties of Constructive Mathematics, Cambridge University Press, Cambridge.

Brouwer, L. E. J. (1927): 'Über Definitionsbereiche von Funktionen', Mathematische Annalen 97: 60-75. English extract in J. van Heijenoort (ed.), From Frege to Gödel: a Source Book in Mathematical Logic, 1897-1931, Harvard University Press, Cambridge, MA, 1967: 457-463.

Dalen, D. van (1973): 'Lectures on Intuitionism', in A. R. D. Mathias and H. Rogers (eds.), Cambridge Summer School of Mathematical Logic, Springer, Berlin: 1-94.

Dalen, D. van (1983): Logic and Structure (2nd. rev. ed), Springer, Berlin.

Dalen, D. van (1986): 'Intuitionistic logic', in D. Gabbay and F. Guenthner (eds.), Handbook of Philosophical Logic, Vol. III, Reidel, Dordrecht: 225-339.

Dummett, M. A. E., with the assistance of R. Minio (1977): Elements of Intuitionism, Clarendon Press, Oxford.

Gentzen, G. (1935): 'Untersuchungen über das logische Schliessen (I, II)', Mathematische Zeitschrift 39: 176-210. Quoted by the English version in the Collected Papers, ed. by M. E. Szabo, North-Holland, Amsterdam, 1969: 69-131.

Heyting, A. (1934): Mathematische Grundlagenforschung: Intuitionism, Beweistheorie, Springer, Berlin. Quoted by the French expanded edition, Les Fondements des Mathématiques. Intuitionnisme. Théorie de la Démonstration, Gauthier-Villars, Paris, 1955.

Heyting, A. (1956): Intuitionism: an Introduction, North-Holland, Amsterdam.

Kolmogorov, A. N. (1932): 'Zur Deutung der intuitionistischen Logik', Mathematische Zeitschrift 35: 58-65.

Kreisel, G. (1961): 'Set-theoretical problems suggested by the notion of potential totality', in Infinitistic Methods: Proceedings of the Symposium of Foundations of Mathematics, Warsaw 2-9 September 1959, Pergamon Press, Oxford: 103-140.

Kreisel, G. (1962): 'Foundations of intuitionistic logic', in E. Nagel, P. Suppes and A. Tarski (eds.), Logic, Methodology and Philosophy of Science, Vol. I, Stanford University Press, Stanford, CA: 198-210.

Kreisel, G. (1965): 'Mathematical logic', in T. L. Saaty (ed.), Lectures on Modern Mathematics, Vol. III, Wiley and Sons, New York: 95-195. 
Kreisel, G. (1970): 'Church's thesis: a kind of reducibility axiom for constructive mathematics', in J. Myhill, A. Kino and R. E. Vesley (eds.), Intuitionism and Proof Theory, North-Holland, Amsterdam: 121-150.

Martin-Löf, P. (1984): Intuitionistic Type Theory: Notes by Giovanni Sambin of a series of lectures given in Padova, June 1980, Bibliopolis, Naples.

Martin-Löf, P. (1985): 'On the meanings of the logical constants and the justifications of the logical laws', in Atti degli Incontri di Lògica Matemàtica, Vol. II, Scuola di Specializzazione in Lògica Matemàtica, Dipartimento di Matemàtica, Università di Siena: 203-281. Quoted by the reprint in Nordic Journal of Philosophical Logic 1 (1996): 11-60.

Martin-Löf, P. (1987): 'Truth of a proposition, evidence of a judgement, validity of a proof', Synthese 73: 407-420.

Martin-Löf, P. (1994): 'Analytic and synthetic judgements in type theory', in P. Parrini (ed.), Kant and Contemporary Epistemology, Kluwer, Dordrecht: 87-99.

Nordström, B., K. Petersson and J. M. Smith (1990): Programming in MartinLöf's Type Theory: an Introduction, Clarendon Press, Oxford.

Prawitz, D. (1977): 'Meaning and proofs: on the conflict between classical and intuitionistic logic', Theoria 43: 1-40.

Sundholm, G. (1983): 'Constructions, proofs and the meaning of the logical constants', Journal of Philosophical Logic 12: 151-172.

Sundholm, G. (1986): 'Proof theory and meaning', in D. Gabbay and F. Guenthner (eds.), Handbook of Philosophical Logic, Vol. III, Reidel, Dordrecht: 471-506.

Sundholm, G. (1994): 'Vestiges of realism', in B. McGuiness and G. Oliveri (eds.), The Philosophy of Michael Dummett, Kluwer, Dordrecht: 137-165.

Troelstra, A. S. (1969): Principles of Intuitionism, Springer, Berlin.

Troelstra, A. S. (1977): 'Aspects of Constructive Mathematics', in K. J. Barwise (ed.), Handbook of Mathematical Logic, North-Holland, Amsterdam: 9731052.

Troelstra, A. S. and D. van Dalen (1988): Constructivism in Mathematics: an Introduction, Vol. I, North-Holland, Amsterdam.

Weinstein, S. (1983): 'The intended interpretation of intuitionistic logic', Journal of Philosophical Logic 12: 57-82. 